\title{
Predictibilidad en el Dianostico usando Inteligencia artificial
}

\author{
Predictability in Diagnosis using Artificial Intelligence \\ Previsibilidade no Diagnóstico com Inteligência Artificial
}

\section{ARTÍCULO GENERAL}

Maria Gabriela Garcia Zuleta

https://orcid.org/0000-0002-7823-1656

Recibido 01 de Diciembre 2020 | Arbitrado y aceptado 26 de Diciembte 2020 | Publicado el 01 de Febrero 2021

\section{RESUMEN}

El presente estudio trata sobre una revisión documental relacionada con la predictibilidad en el diagnóstico utilizando Watson de IBM, el cual tiene como objetivo principal "conocer el nivel de predictibilidad en el diagnóstico con Watson de IMB "se ha utilizado un diseño no experimental de corte cualitativo donde las técnicas de recolección de datos han sido la revisión documental y el análisis de datos provenientes de estudios realizados por expertos que validan el uso de este tipo de tecnología, de acuerdo a los documentos consultados y en función de la opinión de los expertos se puede referir que la hipótesis planteada "el conocer el nivel de predictibilidad en el diagnóstico utilizando Watson de IMB contribuye a su implementación en el diagnóstico de enfermedades de orden oncológico" se cumple y como parte de algunas de las conclusiones las cuales refiere que el uso de este tipo de tecnología aporta a la prontitud en la generación de diagnósticos más certeros y con un nivel de predictibilidad media - alta, al igual que es una oportunidad para el trabajo colaborativo entre profesionales de la salud y representa la necesidad imperante de actualizar sus conocimientos para proporcionar datos de calidad que faciliten una mayor predictibilidad del diagnóstico.

Palabras clave: Predictibilidad, Predictibilidad en diagnóstico, Watson, IBM.
The present study deals with a documentary review related to the predictability in the diagnosis using IBM Watson, which has as its main objective "to know the level of predictability in the diagnosis with IBM Watson". a qualitative nonexperimental design has been used where the data collection techniques have been the documentary review and the analysis of data from studies conducted by experts who validate the use of this type of technology, according to the documents consulted and based on the opinion of the experts, it can be stated that the hypothesis "knowing the level of predictability in the diagnosis with Watson IMB contributes to its implementation in the diagnosis of oncological diseases" is fulfilled and as part of some of the conclusions it is stated that the use of this type of technology contributes to the promptness in the generation of more accurate diagnoses and with a medium-high level of predictability, It is also an opportunity for collaborative work among health professionals and represents the imperative need to update their knowledge in order to provide quality data that facilitate greater diagnostic predictability.

Keywords: Predictability, Predictability in diagnostics, IBM, Watson.

\section{RESUMO}

O presente estudo trata-se de uma revisão documental relacionada à previsibilidade no diagnóstico utilizando o IBM Watson, que tem como objetivo principal "conhecer o nível de previsibilidade no diagnóstico com o IBM Watson". foi utilizado um design qualitativo não experimental onde as técnicas de coleta de dados foram a revisão documental e a análise de dados de estudos realizados por especialistas que validam o uso desse tipo de tecnologia, de acordo com os documentos consultados e com base na opinião de os especialistas, pode-se afirmar que a hipótese "conhecer o nível de previsibilidade no diagnóstico com Watson IMB contribui para sua implementação no diagnóstico de doenças oncológicas" é cumprida e como parte de algumas das conclusões afirma-se que o uso de Esse tipo de tecnologia contribui para a agilidade na geração de diagnósticos mais precisos e com nível médio-alto de previsibilidade, é também uma oportunidade de trabalho colaborativo entre os profissionais de saúde e representa a necessidade imperiosa de atualizar seus conhecimentos para oferecer qualidade dados que facilitam uma maior previsibilidade diagnóstica.

Palavras-chave: Previsibilidade, Previsibilidade em diagnósticos, IBM, Watson. 


\section{Introducción}

En la lucha contra el cáncer cada vez tiene mayor importancia asociar el tratamiento con la huella genética del tumor. De esta forma se mejoran los resultados, pero para esto los médicos necesitan semanas, durante las que tratan de identificar cuál es la mejor medicación para atacar la mutación que está causando el cáncer. Sin embargo, el sistema de computación cognitiva IBM Watson emplea en esta tarea unos pocos minutos.

La supercomputadora de IBM rebusca en su enorme base de datos, repleta de papers científicos, textos de todo tipo y ensayos clínicos sobre diferentes cánceres y sus tratamientos potenciales, con el fin de encontrar la terapia más adecuada en cada caso. El ahorro de tiempo podría resultar providencial, con lo que 14 centros médicos (en Estados Unidos y Canadá) especializados en el tratamiento del cáncer han llegado a un acuerdo para utilizar Watson. (Agrawal, Gans, \& Goldfar, 2018)

Los centros médicos comenzaron a usar la versión cloud del sistema a finales de 2015. El objetivo fue ofrecer a los pacientes tratamientos personalizados a fin de incrementar al máximo las posibilidades de curación.

Este tipo de tecnología concluye qué tratamiento es el adecuado para cada caso, los oncólogos tienen que introducir en el sistema la huella genética del tumor, los datos claves para saber qué genes han mutado, pues posiblemente estos sean los causantes del cáncer. A partir de ahí Watson hace su investigación y propone soluciones a los médicos.

\section{Aspectos teóricos}

$\mathrm{IBM}^{\circledR}{ }^{\circledR}$ Watson ${ }^{\mathrm{TM}}$ representa un primer paso hacia los sistemas cognitivos, una nueva era de la informática (High, 2012). El IBM Watson Analytics es un sistema cognitivo basado en el procesamiento del lenguaje natural, el aprendizaje dinámico, la generación y evaluación de hipótesis. Una vez que el conjunto de datos se ha cargado en la nube, IBM Watson Analytics puede predecir las consultas, asociar los datos y recopilarlos para obtener información. Por objetivo, este sistema proporciona puntuaciones de calidad de datos, análisis detallados y asociaciones de campo (Nagwanshi \& Dubey, 2017) 
Según Archut (2015) Watson Analytics aprovecha sus capacidades cognitivas de tres formas:

- Proporciona reconocimiento semántico de conceptos en sus datos, como tiempo, lugar e ingresos.

- Proporciona puntos de partida recomendados para el análisis y objetivos para la predicción.

- Permite la interacción en lenguaje natural, lo que permite la exploración de datos a través de preguntas sencillas que podría hacerle a un colega.

En la actualidad, un $80 \%$ de los pioneros en IA está invirtiendo en el proceso de entrenamiento de algoritmos (Ransbotham et al. 2018). Así, IBM Watson ${ }^{\circledR}$ representa una ayuda para los científicos de datos y los desarrolladores a acelerar la implementación de IA y machine learning. Con su operación de modelo abierto y extensible, Watson ayuda a las empresas a simplificar y aprovechar la IA a escala en cualquier nube (IBM, s.f).

\section{Lenguaje en IBM Watson}

Se ha visto un cambio en las técnicas de construcción para el procesamiento del lenguaje natural cuando se necesita detalle sobre una precisión limitada. Estas técnicas incorporan mucho más contexto en la evaluación de la pregunta. En ese sentido High (2012) indica este concepto como procesamiento profundo del lenguaje natural, que a veces se denomina Deep Question-Answering (DeepQA) cuando el problema es responder a preguntas en lenguaje natural.

De acuerdo con la fuente anterior IBM Watson se fundamenta en un sistema de Procesamiento Superficial del Lenguaje Natural (PNL o NLP por sus siglas en inglés) porque, aunque podría ser bastante adecuado dentro de su enfoque más estrecho, no es muy preciso.

En ese sentido, IBM Watson alcanza precisión al intentar evaluar tanto contexto como le sea posible. Obtiene ese contexto tanto dentro del pasaje de la pregunta como de la base de conocimientos (llamada corpus) que está disponible para encontrar respuestas. 
Asimismo, el autor señala que IBM Watson es un ejemplo de sistema cognitivo, tal y como se había mencionado previamente. Ello hace posible que pueda separar el lenguaje humano para identificar inferencias entre pasajes de texto con una precisión similar a la humana, y a velocidades y escalas que son mucho más rápidas y mucho más grandes que cualquier otra.

Figura 1. Cómo Watson obtiene una respuesta a una pregunta

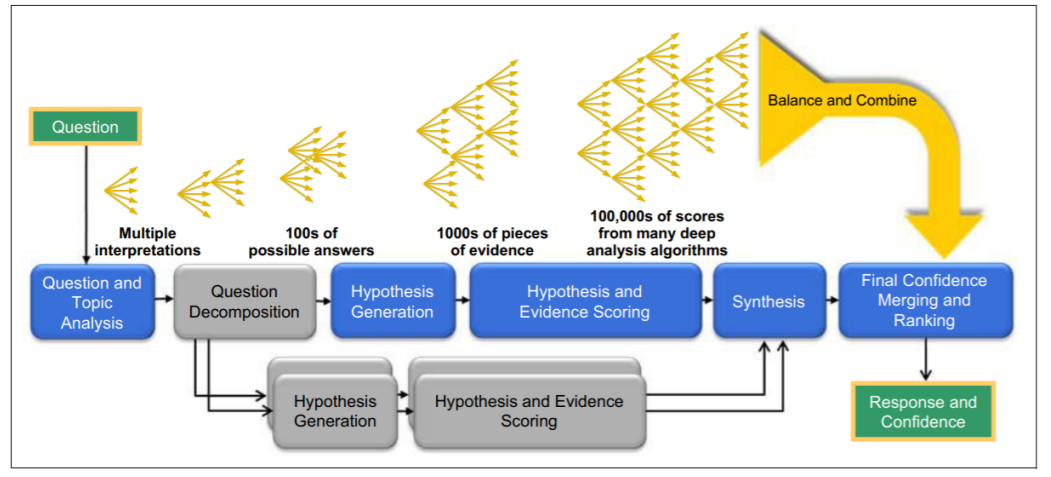

De esta forma, al igual que los humanos, los sistemas cognitivos se ven impulsados a comprender conceptos descomponiendo las expresiones de una idea y luego combinando los resultados con el contexto y la probabilidad de que ciertos términos del pasaje se utilicen de cierta manera. Y, al igual que con los humanos, la confianza es proporcional a la evidencia que respalda esas probabilidades y la cantidad de algoritmos de razonamiento que tenemos disponibles para probar nuestras hipótesis.

\section{Predictibilidad}

La predictibilidad depende de una serie de factores, como la interacción con el subsistema indeterminista, los requisitos de datos de RTT, los recursos y los conflictos de datos entre diferentes RTT de procesamiento, el mecanismo de recuperación tradicional y la coherencia mutua entre los datos replicados (Shrivastava \& Shanker, 2019).

\section{IBM Watson y predictibilidad en diagnósticos}

El uso de las tecnologías de la información (TI) para el cuidado de la salud ya no es un concepto nuevo. El uso de computadoras y TI en todos los campos se ha convertido en una práctica común. El registro de historiales de pacientes, detalles, facturación y otra información en computadoras se ha convertido en una práctica común en todas partes (Aggarwal \& Madhukar, 2020). 
Así, en el ámbito de la salud, los proveedores, los líderes y los investigadores de cuidado médico están utilizando las tecnologías de IA, como IBM Watson, para aprovechar los millones de informes médicos, los registros de pacientes, los ensayos médicos y las revistas médicas para descubrir insights de datos (Krishnan, 2020).

IBM Watson Health Cloud for Life Sciences Compliance e IBM Watson Care Manager se lanzó en 2015. Tiene fusiones con la Universidad de Columbia, el Boston Children's Hospital, ICON plc, Teva Pharmaceuticals y Sage Bionetworks. En 2015, la adquisición de Merge Healthcare Inc., una empresa líder que ayuda a los médicos a almacenar y acceder a imágenes médicas, supone un gran avance para Watson Health Cloud. (Macmillan \& Dowskin, 2015). En la actualidad, IBM Watson puede integrarse con cualquier problema médico, el usuario puede crear sus propias aplicaciones de IoT para cualquier enfermedad que desee, ya que Watson no impone restricciones. Más recientemente, IBM se ha unido a Apple, por ejemplo, para predecir el estado de salud en función del hábito de dormir.

Se ha encontrados como parte de la revisión documental que efectivamente ¿Cómo hace Watson para analizar una consulta?

- Descompone la consulta mediante análisis sintácticos/semánticos en frases con información relevante. Mediante algoritmos de diversos tipos buscan las parafrases, elementos de tipo geográfico, establece equivalencias de contenido, etc

- La mayor preocupación es que la actividad se realiza en tiempo real y para tomar la decisión se realizan procesos paralelos para construir posibles respuestas tan grande como sean posibles.

- Algo importante es lo que queremos construir entre el Plano de la ideación del proceso y el Plano de la realidad; ya que hay que construir patrones/soluciones que permitan aplicar esta tecnología.

\section{Utilizaciones a las que se pueden aplicar:}

- Consultor (Engagement Advisor): Como sistema que da soporte a una persona, a partir de una consulta en lenguaje natural. Ejemplo. Contact Center 
- Asesor (Discovery Advisor): Plantea una respuesta a partir de relacionar una variada información relevante. Ejemplo: Buscar fármacos que tengan sustancias químicas determinadas.

- Asesor oncológico (Oncology Advisor): Sistema que ayuda a la recomendación del tratamiento en escenarios que tengan las características del paciente y, que le permitan mejorar su calidad de vida. La recomendación toma en cuenta el Historial médico del paciente y tratamientos oncológicos anteriores (históricos) conservados en la plataforma.

- Asesor de control (Police Advisor): Si algo relevante en lo que estamos trabajando se adecua o no se adecua a un cuerpo, a la hora de tomar decisiones.

\section{¿Cómo activamos Watson?}

- Cargar información del dominio (Corpus)

- Ingresar toda la información relevante del dominio

- Limpiar el contenido de la información

- Eliminar información obsoleta, contradictoria, etc

- Entrenarlo con preguntas

- Hay que usar a los expertos para que carguen preguntas/ respuestas del negocio

(dominio concernido)

- Debe usarse aproximadamente 800 preguntas para un Prototipo y tener 3,000 para un sistema listo para pase a producción

- Esto toma aproximadamente entre 6-8 meses que son pequeños en tiempo pero intensos en esfuerzos

- Probar el sistema

- Iterar entrenamiento

\section{Acuerdo entre los actores del mercado}


En Estados Unidos, los grandes jugadores más tradicionales en el sector salud (entre los que se destacan GE, Philips, Siemens) como así también los nuevos participantes (principalmente Google, IBM, Microsoft) han comenzado a realizar acuerdos estratégicos con instituciones académicas con el objetivo de contar con el acceso a datos a cambio de su financiamiento en investigación. Por otro lado, dado que la solución que realiza cada empresa suele atacar un caso de uso específico, se han comenzado a realizar acuerdos entre compañías para ampliar la oferta de servicios al cliente (Harvey, 2017).

\section{Configuración del mercado}

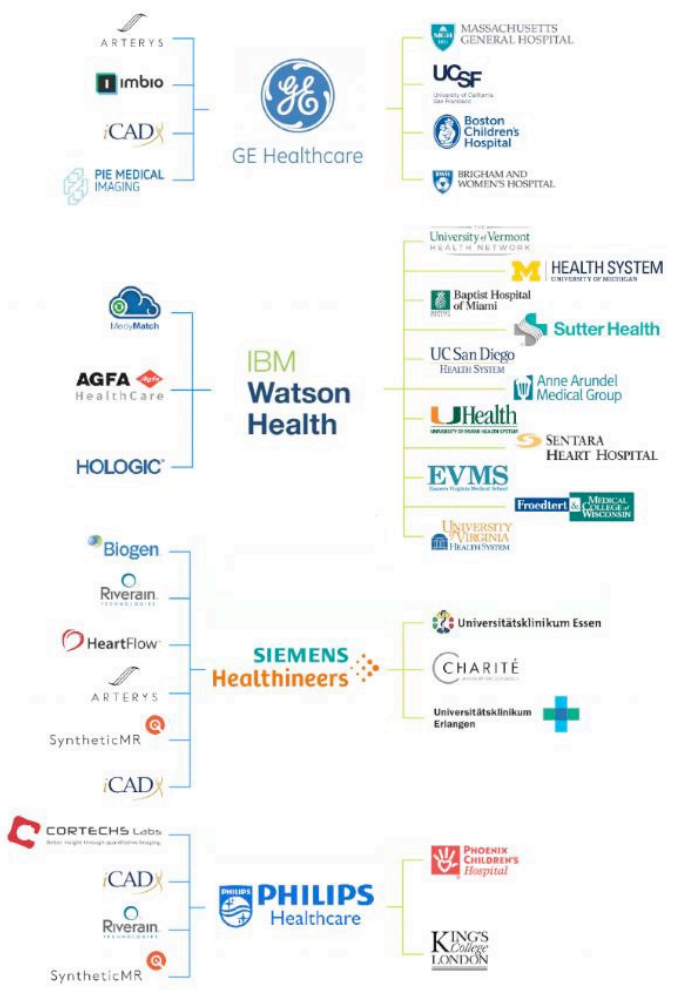

Ejemplos de integración de grandes compañías (GE, IBM, Siemens, Philips) con Centros de salud y startups. Fuente: UK can lead in Radiology AI. Here's how... (Harvey, 2017).

\section{Metodología}

El trabajo llevado a cabo estará principalmente caracterizado por una metodología de tipo exploratoria la cual de acuerdo a un tipo de investigación utilizada para estudiar un problema que no está claramente definido, por lo que se lleva a cabo para 
comprenderlo mejor, pero sin proporcionar resultados concluyentes (Hernández Sampieri, Fernández Collado, \& Pilar, Metodología de la investigación, 2018)

En referencia a los trabajos exploratorios, Dankhe (1986) agrega que, generalmente, no son un fin en sí mismo, sino que "...por lo general determinan tendencias, identifican relaciones potenciales entre variables y establecen el "tono" de investigaciones posteriores más rigurosas". En este trabajo se está analizando la predictibilidad en el diagnóstico de Watson de IBM que, si bien, viene siendo estudiado desde una perspectiva médica-científica para casos puntuales, es muy escasa desde un punto de vista de management, limitándose a reportes de fuentes no académicas.

El paradigma de nuestro trabajo será cualtitativo, y como tal, se pondrá en práctica algunos de sus métodos, como son el análisis de fuentes primarias y secundarias, entrevistas en profundidad y la observación (Cook \& Reichardt, 1986).

\section{Preguntas de investigación}

¿Cuál es el nivel de predictibilidad en el diagnóstico utilizando Watson de IMB?

¿Existe una industria que actualmente impulse este tipo de tecnología y de soporte a los resultados?

¿Puede Machine Learning mejorar la precisión de resultados?

\section{Objetivos de la investigación}

\section{General}

Conocer el nivel de predictibilidad en el diagnóstico utilizando Watson de IMB

\section{Específicos}

- Describir el avance de la industria que impulsa este tipo de tecnología y da soporte a los resultados

- Explicar como puede la Machine Learning mejorar la precisión de resultados 


\section{Hipótesis}

\section{General}

El conocer el nivel de predictibilidad en el diagnóstico utilizando Watson de IMB contribuye a su implementación en el diagnóstico de enfermedades de orden oncológico.

\section{Específicas}

El uso de AI permite el desarrollo de nuevas tecnologías que respaldan la precisión de los resultados utilizando por Watson de IBM

La implementación de Watson de IMB favorece el tiempo y el costo de atención de los pacientes.

\section{Diseño}

El diseño de la investigación son estrategias o planes concebidos para obtener la información deseada; en ese sentido, los diseños pueden ser, experimentales y no experimentales, la presente investigación es no experimental, ya que esta es aquella que se realiza sin manipular deliberadamente variables. Se basa fundamentalmente en la observación de fenómenos tal y como se dan en su contexto natural para analizarlos con posterioridad (Hernández Sampieri, Fernández Collado, \& Pilar, Metodología de la investigación, 2018)

\section{Técnicas}

Se ha planificado trabajar con técnicas cualitativas como la revisión documental

La revisión documental es una técnica de observación complementaria, en caso de que exista registro de acciones y programas. La revisión documental permite hacerse una idea del desarrollo y las características de los procesos y también de disponer de información que confirme (Hernández Sampieri, Fernández Collado, \& Pilar, Metodología de la investigación, 2018).

\section{Población y muestra}

Por ser un tipo de estudio exploratorio se contado con la opinion de al menos 5 expertos que han utilizado este tipo de tecnología para predecir los resultados en los 
pacientes. Igualmente se realizó la revisión documental de información basada en papers de los últimos tres años.

\section{Procedimientos y análisis de los datos}

La información acopiada, se ha sometido a un proceso codificación de datos de las variables y de la información acopiada que corresponde tanto a la variable independiente como dependiente.

\section{Resultados y discusión}

1. Daniel Tse et al. (2019 a realizado la detección de nódulos pulmonares de diferente tamaño y solidez donde ocurrió una disminución del 11\% en informes con falsos positivos y una disminución de $5 \%$ de falsos negativos, lo cual a su vez se puede contratar con lo establecido por Yasaka \& Abe, 2018 quienes han establecido la relación de este tipo de AI donde se genera mayor información detallada con cortes cada vez más finos.

2. Según Selanikio, (2018) Watson por Oncology es un desarrollo que toma como entrada la información de pacientes (laboratorios, historia clínica, entre otros), y la contrasta con literatura médica y otra información con el fin de realizar recomendaciones para el tratamiento lo cual se compara con las apreciaciones de Larusso (2019) gastroenterólogo de Mayo Clinic y director del proyecto de colaboración entre Mayo y el sistema Watson de IBM. "Al contar con menores tiempos desde el inicio hasta la compleción de los ensayos, nuestros equipos de investigación podrán realizar estudios más profundos y completos", comenta el “Además de la mayor exactitud, podremos desarrollar, refinar y mejorar técnicas nuevas y más convenientes a un nivel mayor en medicina"

3. Según Max Tegmark, (2017) Watson fue concebida para responder preguntas, haciendo uso de PLN, IA, representación de conocimiento, razonamiento automatizado y ML. Luego, sus formas de uso se fueron ampliando lo cual se compara en la práctica con lo expuestos por Alberts (2017) director de oncología médica en Mayo Clinic "En un área como el cáncer, donde el tiempo es fundamental, la velocidad y exactitud del sistema Watson permite desarrollar más 
eficientemente un plan de tratamiento personalizado para ofrecer al paciente la atención exacta que requiere".

4. Legrand (2017) menciona que Watson es una herramienta que tiene por objetivo crear un registro de paciente más confiable y mejorar la calidad de reportes y procesos lo cual al compararse su implementación menciona Wang, (2016) que el diagnóstico temprano y la toma de decisiones pueden recortar los costos a más del $50 \%$. Aproximadamente el $20 \%$ de los costos asociados al diagnóstico son salarios, por lo que podría haber un gran ahorro si la IA mejora la eficiencia de los médicos y otros profesionales de la salud.

5. Stumpe, (2017) establece que en patología al realizar análisis de cáncer de mama y de prostata se ha encontrados hasta u nivel de predictibilidad de $73 \%$ promedio para un patólogo sin limitaciones de tiempo, esto a su vez puede ser contrastado con lo expuestos por Roach, (2016), quien estableció que patólogos para algunas formas de cáncer de mama puede llegar a ser menor al 48\%, de igual forma que para el cáncer de prostata lo cual evidencia que efectivamente el sistema Watson elimina el tiempo de predicción convencional lo cual beneficia al paciente para el inicio de su tratamiento.

\section{Conclusiones}

El sistema Watson para el diagnóstico de procesos oncológicos se ha encontrado como una solución esperanzadora para la agilización y precisión del cáncer lo cual su vez les permite a los pacientes contar con un tratamiento más oportuno y orientado a solucionar su problema de salud.

Este tipo de sistemas representa para los profesionales de la salud, la oportunidad de actualizarse y apoyar a los pacientes en la dura carrera por cuidar y estimar la salud en el caso de las enfermedades de orden oncológico.

La implementación de este tipo de sistemas igualmente exige el trabajo de carácter multidisciplinario, dado que de esta forma se logran optimizar los resultados en cada una de las áreas en las cuales se ha probado Watson, los resultados obtenidos utilizando este tipo de tecnología colabora con el profesional de la salud en dictaminar diagnósticos más certeros. 
Para las entidades de salud, la inversión inicial en el uso de estos equipos es alto sin embargo si se realiza una comparativa de los ahorros que presentan en cuanto a los procesos de diagnóstico, atención y hospitalización de los pacientes es bastante aceptable y a esto se suma la carga horaria que deben actualmente dedicar médicos, enfermeras y especialistas de la salud en general para atender enfermedades de orden oncológico.

El uso de este tipo de tecnología representa la transferencia de valor generada entre los distintos actores del sistema de salud, ya que la AI está creciendo y está siendo enfocada hacia nuevas áreas de la salud.

\section{Referencias}

Albert, S. (2017). Informe de aplicabilidad de Watson en el área oncológica de clínica Mayo.

Aggarwal, M. \& Madhukar, M. (2020) IBM'S Watson Analytics for Health Care: A Miracle made true. Cloud Computing Systems and Applications in Healthcare. https://www.researchgate.net/publication/316202671 IBM's Watson Analytics f or Health_Care

Archut, S. (2015) Get the facts on IBM Watson Analytics. IBM Big Data \& Analytics Hub.

Bernal. (2012). Metodología de la investigación (Tercera ed.). Bogotá, Colombia: Pearson Educación. Recuperado el 16 de febrero de 2021

Cook, T. D., Reichardt, C. S., Manuel, J., \& Guillermo (trad.) Solana. (1986). Métodos cualitativos y cuantitativos en investigación evaluativa. Madrid: Morata.

Dankhe, G. (1986). Definición de Investigación. En G. Dankhe, Metodología de la investigación.

Hernández Sampieri, R., Fernández Collado, C., \& Pilar, B. L. (2018). Metodología de la investigación. México: McGraw-Hill Interamericana.

High, R. (2012) The Era of Cognitive Systems: An Inside Look at IBM Watson and How it Works. IBM Corp, New York. http://www.redbooks.ibm.com/redpapers/pdfs/redp4955.pdf 
IBM (s.f) IBM Watson Machine Learning. https://www.ibm.com/pe-es/cloud/machinelearning

Krishnan, A. (2020) How well-trained, mature AI solutions can earn trust. Watson Health Perspectives - IBM. https://www.ibm.com/blogs/watson-health/well-trainedmature-ai/

Larusso, N. (2019). Efectividad en el uso de Watson en clínica Mayo.

Legrad, G. (2017). The Definition of Health. Towards New Perspectives, 1(14). doi:https://doi.org/10.1177/0020731418782653

Macmillan, R. \& Dowskin, E. (2015). IBM Crafts a Role for Artificial Intelligence. Wall Street Journal. https:/www.wsj.com/articles/ibm-crafts-a-role-for-artificialintelligence-in-medicine-1439265840

Nagwanshi \& Dubey (2017) Statistical Feature Analysis of Human Footprint for Personal Identification Using Big ML and IBM Watson Analytics. Arabian Journal for Science and Engineering, Issue 6. DOI 10.1007/s13369-017-2711-z

Ransbotham, S. Gerbert, P., Reeves, M., Kiron, D. \& Spira, M. (2018) Artificial intelligence in business gets real: Pioneering Companies Aim for AI at Scale. MITSloan. https://sloanreview.mit.edu/projects/artificial-intelligence-in-businessgets-real/

Roach, J. (2016). Microsoft researchers detect lung-cancer risks in web search logs. Obtenido de Microsoft researchers detect lung-cancer risks in web search logs: https://blogs.microsoft.com/ai/microsoft-researchers-detect-lung-cancer-risksweb-search-logs/

Shrivastava, P. \& Shanker, U. (2019) Supporting Transaction Predictability in Replicated DRTDBS. ICDCIT, pp. 125-140, 2019. https://doi.org/10.1007/978-3-030-05366$\underline{6} 10$

Selanikio, J. (2018). The Shaming of Watson. Medium - Blog - Becoming Human. Retrieved from. Obtenido de https://becominghuman.ai/the-shaming-of-watson$\underline{3 \mathrm{~d} 0672 \mathrm{~d} 59 \mathrm{f} 1}$ 
Stumpe, M. (2017). Assisting Pathologists in Detecting Cancer with Deep Learning. Obtenido de Assisting Pathologists in Detecting Cancer with Deep Learning: https://ai.googleblog.com/2017/03/assisting-pathologists-in-detecting.html

Tegmark, M. (2017). Vida 3.0 Tecnología médica.

Tse, D. (2019). Imagine your world with Watson. Treatment and differential diagnosis insights for thephysician's consideration in the moments that matter most. Obtenido de Imagine your world with Watson. Treatment and differential diagnosis insights for thephysician's consideration in the moments that matter most: https://www.ibm.com/blogs/watson-health/wp-content/uploads/2016/12/WHI-

\section{Overview-Executive-Brief.pdf}

Wang, J. (2016). ARK Invest - Deep Learning Based Diagnostics:. doi:https://arkinvest.com/research/deep-learning-based-diagnostics

Yasaka, K., \& Abe, O. (s.f.). Deep learning and artificial intelligence in radiology: Currentapplications and future directions. PLOS Medicine, 15(11). doi:https://doi.org/10.1371/journal.pmed.1002707 